\title{
EFICIÊNCIA DE FOSFITOS NO CONTROLE DA PODRIDÃO DA BASE DO ESTIPE EM MUDAS DE PUPUNHEIRA ${ }^{1}$
}

\author{
EDUARDO JUN FUZITANI ${ }^{2}$, ÁLVARO FIGUEREDO DOS SANTOS ${ }^{3}$, \\ WILSON DA SILVA MORAES ${ }^{2}$, ERVAL RAFAEL DAMATTO JUNIOR ${ }^{2}$, \\ EDSON SHIGUEAKI NOMURA ${ }^{2}$
}

RESUMO- A exploração de palmito no Brasil está deixando de ser uma atividade extrativista para tornarse agrícola, devido ao plantio de palmeiras, como a pupunheira (Bactris gasipaes Kunth var. gasipaes Henderson). A podridão da base do estipe (PBE), causada por Phytophthora palmivora, vem destacando-se como uma importante doença, que poderá limitar o cultivo da pupunheira em várias regiões produtoras. Esta doença ocorre em plantas jovens e adultas. O objetivo do presente trabalho foi avaliar a eficiência da aplicação preventiva e curativa de fosfitos sobre a incidência e a severidade da PBE em mudas de pupunheira, em casa de vegetação. Para tal, três experimentos foram conduzidos, conforme segue: I - Efeito preventivo e curativo de fosfitos, seguindo o esquema fatorial 6 × 2 (tipos de fosfitos x épocas de aplicação); II - Efeito da aplicação preventiva de fosfito de potássio, seguindo o esquema fatorial 6 x 4 (número de aplicações $\mathrm{x}$ épocas de avaliação); e III - Efeito preventivo do número de aplicações e dosagens de fosfitos de potássio, seguindo o esquema fatorial 3 x 2 (dosagens x número de aplicações). A severidade da doença foi avaliada aos 28 dias no experimento I e no experimento III, enquanto no experimento II foi avaliada aos 7; 14; 21 e 28 dias após a inoculação, baseada em escala descritiva, com nota de 0 a 4 . Os tratamentos foram dispostos no delineamento inteiramente casualizado, com 10 repetições de uma muda cada. Os dados foram submetidos à análise de variância e, quando significativos, pelo teste $\mathrm{F}(\mathrm{p} \leq 0,05)$, e as médias dos tratamentos foram comparadas pelo teste de Tukey, a 5\% de probabilidade. Pelos resultados obtidos, pôde-se concluir que o fosfito de potássio proporcionou melhor controle entre os fosfitos testados, e quando aplicado de forma preventiva na dosagem de 2,5 mL. $\mathrm{L}^{-1}$ com 3 aplicações e na dosagem de 5,0 mL.L $\mathrm{L}^{-1}$ com 2 ou 3 aplicações, reduziu significativamente a incidência e a severidade da PBE em mudas de pupunheira. Entretanto, quando aplicado de forma curativa, não teve efeito, independentemente da dosagem ou do número de aplicações. A melhor época de avaliação da severidade da PBE foi aos 21 dias após a inoculação da Phytopthora palmivora. Termos para indexação: Bactris gasipaes Kunth; pupunha; palmito; Phytophthora palmivora; fitopatologia.

\section{PHOSPHITES EFFICIENCY ON PEACH PALM STEM BASE ROT CONTROL}

ABSTRACT - The exploitation of heart of palm in Brazil is no more an extractive activity; it is becoming a commercial production due to the planting of palm trees such as peach palm (Bactris gasipaes Kunth var. gasipaes Henderson). The stem base rot (SBR) caused by Phytophthora palmivora has emerged as an important disease, which may limit the peach palm production in many regions. This disease occurs in young and adult plants. This study aimed to evaluate the efficiency of preventive and curative application of phosphites on SBR incidence and its severity in peach palm seedlings under greenhouse conditions. Three experiments were carried out, as follows: I - preventive and curative effect of phosphites, following factorial scheme $6 \times 2$ (phosphites x application time); II - effect of preventive application of potassium phosphite, following factorial scheme 6 x 4 (number of applications x evaluation periods), and III - Effect of preventive and curative methods and application periods of potassium phosphite, following a factorial scheme $2 \times 2$ (application forms $\mathrm{x}$ dosage). Disease severity was assessed at 28 days in experiment I and III in the experiment, while in experiment II was evaluated at 7, 14, 21 and 28 days after inoculation, based on descriptive scale with a scale from 0 to 4 . Treatments were carried out in a completely randomized design with 10 repetitions of one seedling for each repetition. Data were submitted to variance analysis and when treatment means were significant by $F$ test $(\mathrm{p} \leq 0.05)$ they were compared by Tukey test at $5 \%$ of probability. From the results obtained, it was concluded that potassium phosphite have better control between phosphites tested, and when applied preventively at $2.5 \mathrm{~mL} . \mathrm{L}^{-1}$ with 3 applications and dosage of $5.0 \mathrm{~mL}$. L $\mathrm{L}^{-1}$ with 2 or 3 applications significantly reduced the incidence and the severity of SBR in peach palm seedlings. But, when applied as curative had no effect, regardless of the dose or the number of applications. Since the best time for severity assessment of SBR was 21 days after inoculation of Phytopthora palmivora.

Index terms: Bactris gasipaes Kunth; peach palm; heart of palm; Phytophthora palmivora;

\footnotetext{
1(Trabalho 234-12). Recebidoe em: 28-08-2012. Aceito para publicação em: 01-11-2010.

${ }^{2}$ Pesquisadores Científicos da APTA, Regional do Vale do Ribeira. - Rod. Régis Bittencourt, km 460, Cx. P:122, Cep: 11900-000, Registro/SP. E-mail: edufuzitani@apta.sp.gov.br,wilson@apta.sp.gov.br, erval@apta.sp.gov.br; edsonnomura@apta.sp.gov.br ${ }^{3}$ Pesquisador Embrapa Florestas - Estrada da Ribeira, Km 111,Colombo/PR. E-mail: alvaro@cnpf.embrapa.br
} 


\section{phytopathology·INTRODUÇÃO}

A pupunheira (Bactris gasipaes Kunth. var. gasipaes Henderson) é uma palmeira nativa da região tropical das Américas, com ampla distribuição geográfica, estendendo-se nas regiões noroeste de Honduras, planalto central Boliviano, Guianas, costa do Pacífico, Equador, norte do México e algumas ilhas do Caribe (MORA-URPI et al., 1997). A planta é de múltiplo uso, tendo como principais utilidades, a produção de palmito e de frutos comestíveis ou, ainda, a fabricação de ração de excelente valor nutritivo (MORA-URPI et al., 1997). A podridão da base do estipe (PBE), causada por Phytophthora palmivora (Butler) Butler), incide em mudas, plantas jovens, de 6 a 12 meses, e em plantas adultas de pupunheira. As plantas atacadas caracterizam-se pela murcha e amarelecimento da folha bandeira, seguido de necrose e seca das demais folhas, podendo provocar a morte da planta-mãe e, às vezes, dos perfilhos da touceira. Em cortes longitudinais e transversais na base do caule ou estipe, observa-se o escurecimento dos tecidos internos e uma podridão generalizada (SANTOS et al., 2004).

$\mathrm{O}$ fosfito $\left(\mathrm{PO}_{3}\right)$ atua como fonte nutricional para as plantas, pois quando ele é oxidado a fosfato $\left(\mathrm{PO}_{4}\right)$, passa a ser uma forma assimilável para os vegetais. Estes produtos apresentam atividades antifúngicas que podem atuar diretamente sobre os fungos ou ativar mecanismos de resistência (BLUM et al., 2007; ARAÚJO et al., 2008; GENTILE et al., 2009).

Existem trabalhos demonstrando que o fosfito possui ação fungicida contra doenças e, ao ser combinado com outros elementos, como K, $\mathrm{Ca}, \mathrm{Mg}$ e $\mathrm{Zn}$, pode ter ação fungicida na planta contra patógenos. Atualmente, são usados fosfitos no controle de doenças, causados por oomicetos do gênero Phytophthora e podem contribuir para o controle de doenças de pós-colheita (DIANESE et al., 2009; BLUM et al., 2007 ). Outros relatos reforçam a ideia de que fosfitos e seus correlatos tiveram eficiência comprovada no controle de doenças causadas por Phytophthora spp. em cultivos de mamoeiro (DIANESE et al., 2009). Estudos são necessários para se determinar o período de tempo que o fosfito protege a planta contra a invasão pelo patógeno e o entendimento mais completo da relação entre o patógeno e o hospedeiro, sob a influência dos fosfitos (ARAÚJO et al., 2008; DIANESE et al., 2008). Contudo, o presente estudo constitui um dos primeiros trabalhos que abordam o controle da PBE com o uso de fosfitos no manejo em mudas de pupunheira.
Devido à falta de informações sobre o controle da PBE da pupunheira e do efeito da aplicação preventiva e curativa dos fosfitos neste patossistema, o presente trabalho teve por objetivo avaliar a eficiência, as doses e o número de aplicações de diferentes fosfitos no controle da PBE em mudas de pupunheira, em casa de vegetação.

\section{MATERIAL E MÉTODOS}

Para atingir os objetivos propostos, três experimentos foram desenvolvidos em casa de vegetação do Laboratório de Patologia Florestal da Embrapa Florestas, Colombo-PR, sendo: Experimento I - Efeito preventivo e curativo de fosfito de potássio, fosfito de cálcio, fosfito de zinco, fosfito de magnésio e fosfito de manganês sobre a incidência e a severidade da PBE em mudas de pupunheira. Experimento II - Efeito do número de aplicações preventivo de fosfito de potássio e a época de avaliação. Experimento III - Efeito preventivo de dosagens e do número de aplicações de fosfito de potássio.

Mudas de pupunheira de oito meses de idade, com cerca de $30 \mathrm{~cm}$ de altura (medido com uma régua graduada da base do colo até a primeira inserção da folha), e 3 a 4 pares de folhas, foram mantidas em sacos de polietileno preto $(11 \mathrm{~cm}$ de altura e $7 \mathrm{~cm}$ de largura), contendo subsolo, e irrigadas manualmente de acordo com as necessidades das mudas.

Isolados de $P$. palmivora pertencentes à coleção da Embrapa Florestas foram mantidos por meio de repicagens periódicas em meio de batata-dextrose-ágar (BDA: infusão de $200 \mathrm{~g}$ de batata, $15 \mathrm{~g}$ de dextrose, $17 \mathrm{~g}$ de ágar e $1.000 \mathrm{~mL}$ de água destilada). Para obtenção de zoósporos, as culturas de P. palmivora foram repicadas para placas de Petri contendo meio de cultivo cenoura-ágar (CA: infusão de $200 \mathrm{~g}$ de cenoura, $17 \mathrm{~g}$ de ágar e $1.000 \mathrm{~mL}$ de água destilada esterilizada), mantidas por sete dias, sob luz fluorescente constante em ambiente de laboratório. Ao final deste período, seis mililitros de água destilada e esterilizada foram adicionados a cada placa de Petri, sendo colocadas em geladeira por 30 minutos e, depois, transferidas para a temperatura ambiente por 30 minutos, para a liberação dos zoósporos. As suspensões de zoósporos obtidas em cada placa foram vertidas em béquer para a determinação de sua concentração em hemocitômetro.

O método de injeção da suspensão de zoósporos na base do estipe das mudas de pupunheira, desenvolvido no Laboratório de Patologia Florestal da Embrapa Florestas, Colombo-PR, foi utilizado 
em todos os experimentos. Para tal, utilizou-se de uma seringa descartável com agulha hipodérmica nas dimensões de 1,20 × $40 \mathrm{~mm}$, contendo uma alíquota de $0,1 \mathrm{~mL}$ da suspensão de zoósporos/planta, na concentração de 3,75 x $10^{6}$ no Experimento I; de 2,9 x $10^{7}$ no Experimento II, e de 2,05 x $10^{6}$ zoósporos/ $\mathrm{mL}$ no Experimento III.

O Experimento I foi constituído de 12 tratamentos, instalado em delineamento inteiramente casualizado e esquema fatorial 6 × 2 (fosfitos $\mathrm{x}$ formas de aplicação), com 10 repetições de uma muda cada, utilizando-se de fosfito de potássio $\left(40 \% \mathrm{P}_{2} \mathrm{O}_{5}+20 \%\right.$ $\left.\mathrm{K}_{2} \mathrm{O}\right)$; fosfito de cálcio $\left(30 \% \mathrm{P}_{2} \mathrm{O}_{5}+6 \% \mathrm{Ca}\right)$; fosfito de zinco $\left(40 \% \mathrm{P}_{2} \mathrm{O}_{5}+10 \% \mathrm{Zn}\right)$; fosfito de magnésio $\left(30 \% \mathrm{P}_{2} \mathrm{O}_{5}+4 \% \mathrm{Mg}\right)$, e fosfito de manganês $(30 \%$ $\mathrm{P}_{2} \mathrm{O}_{5}+9 \% \mathrm{Mn}$ ), além do tratamento-testemunha (água), e as formas de aplicação: preventiva (48 horas antes da inoculação) e curativa (48 horas após a inoculação). Todas as soluções dos fosfitos foram aplicadas na concentração de 2,5 mL.L-1, com auxílio de pulverizador manual de $25 \mathrm{~mL}$ de volume.

O Experimento II foi constituído de 24 tratamentos, instalado em delineamento inteiramente casualizado e esquema fatorial 6 × 4 (número de aplicações x épocas de avaliação), com 10 repetições de uma muda cada, sendo o número de aplicações aos: 6; 4 e 2 dias antes da inoculação; 6 e 4 dias antes da inoculação; 4 e 2 dias antes da inoculação; 2 dias antes da inoculação; no mesmo dia da inoculação; inoculação e pulverização no mesmo dia, somente com água. O fosfito de potássio foi aplicado na concentração de 5,0 mL.L-1, com auxílio de pulverizador manual de $25 \mathrm{~mL}$ de volume.

O Experimento III foi instalado em delineamento inteiramente casualizado, em esquema fatorial 3 × 2 (dosagens x número de aplicações), todos com 10 repetições de uma muda cada, sendo: D1 - dose de 2,5 mL.L-1 com 2 aplicações aos 4 e 6 dias antes da inoculação e 3 aplicações aos 2; 4 e 6 dias antes da inoculação (preventivas); D2 - dose de 5,0 mL.L-1 com 2 aplicações aos 4 e 6 dias antes da inoculação e 3 aplicações aos 2; 4 e 6 dias antes da inoculação (preventivas), e D0- tratamentotestemunha com aplicação somente com água. Foi testado apenas na forma de aplicação preventiva, visto que, na forma curativa no experimento I, não se obteve controle da PBE em mudas de pupunheira.

$\mathrm{O}$ fosfito de potássio foi aplicado nos tratamentos na forma de pulverização, com auxílio de pulverizador manual de $25 \mathrm{~mL}$ de volume.

A severidade da PBE de todos os experimentos foi avaliada aos 28 dias após a inoculação no experimento I e experimento III, e aos 7;14;21 e 28 dias no experimento II, com base na escala descritiva desenvolvida no Laboratório de Patologia Florestal da Embrapa Florestas, Colombo-PR, para atingir os objetivos do presente trabalho, cujas notas variaram de 0 a 4, em que: 0 - sem sintomas; 1 - folha bandeira e/ou primeira folha aberta com murcha ou amarelecimento; 2 - folha bandeira, primeira e segunda folhas com murcha ou amarelecimento; 3 - todas as folhas com murcha, amarelecimento ou secas; e 4 - planta morta. A severidade da PBE também foi determinada até 28 dias após a inoculação, pela área abaixo da curva de progresso da doença (AACPD) (CAMPBELL.; MADDEN, 1990), e a incidência foi obtida pela quantificação de plantas com sintomas.

Os dados relativos à severidade da PBE dos três experimentos foram submetidos à análise de variância, e as médias, comparadas pelo teste de Tukey, a 5\% de probabilidade, utilizando-se do programa SISVAR (FERREIRA, 2003). Para o cálculo da área abaixo da curva de progresso da doença, utilizou-se do programa AACPD do projeto CanaSis.

\section{RESULTADOS E DISCUSSÃO}

Aos 28 dias após inoculação das mudas de pupunheira, avaliando-se o Experimento I, detectouse que houve interação entre os fatores (tipos e formas de aplicação dos fosfitos), sendo que, quando se aplicaram preventivamente os fosfitos, foi observado que o fosfito de potássio controlou de forma mais eficaz a severidade da PBE, apresentando nota na escala descritiva de 3,2 , resultado semelhante aos fosfitos de magnésio e de manganês, com nota média de 3,8 . A testemunha resultou em menor controle da PBE $(4,0)$, bem como os fosfitos de zinco $(4,0)$ e cálcio $(3,9)$, os quais tiveram controle semelhante aos fosfitos de magnésio $(3,8)$ e manganês $(3,8)$. Os fosfitos aplicados de forma curativa não demonstraram controle diferenciado para a PBE (Tabela 1).

Apenas o fosfito de potássio controlou de forma diferenciada a PBE quando aplicado em diferentes formas de aplicação, sendo que aplicado preventivamente foi superior no controle da doença. Os demais fosfitos não apresentaram efeitos significativos em função do modo de aplicação, se preventivo ou curativo (Tabela 1).

No trabalho proposto, foram testados cinco tipos de fosfito, sendo que o fosfito de potássio, aplicado preventivamente sobre mudas de pupunheira, obteve melhor controle na incidência e na severidade da PBE. O fosfito de potássio tem sido utilizado no manejo de doenças de plantas, 
principalmente aquelas causadas por oomicetos, como Phytophthora (GENTILE et al., 2009) e por patógenos não pertencentes ao grupo dos oomicetos (DIANESE et al., 2009; DIANESE et al., 2008) ). Com os resultados obtidos no presente trabalho, é possível afirmar que a aplicação na forma preventiva de determinada quantidade deste fosfito, antes da infecção pelo patógeno, pode contribuir para a minimização dos danos causados pela doença em mudas de pupunheira.

Daniel e Guest (2006) demonstraram que a aplicação de fosfito de potássio em Arabidopsis thaliana favoreceu a produção de agregados citoplasmáticos e de fenóis ao redor das células infectadas por $P$. palmivora, reduzindo rapidamente a intensidade da doença, o que foi observado entre 6 e 24 horas após a aplicação, indicando que a aplicação curativa foi eficiente, enquanto no presente trabalho foi verificado que a aplicação preventiva com fosfito de potássio foi mais eficiente no controle da PBE em mudas de pupunheira.

No experimento II, verificou-se que houve interação entre os fatores (número de aplicações x épocas de avaliação). Aos 7 dias de avaliação, não houve diferença significativa entre os tratamentos testados; entretanto, aos 14 dias, os tratamentos $\mathrm{T}_{1}$ (3 aplicações aos 6; 4 e 2 dias antes da inoculação), e $\mathrm{T}_{2}$ (2 aplicações aos 6 e 4 dias antes da inoculação) apresentaram menor severidade da PBE, possivelmente devido ao maior tempo de ação do fosfito sobre o agente causal da doença, pois as mudas tratadas com $\mathrm{T}_{1} \mathrm{e} \mathrm{T}_{2}$ ficaram protegidas com o fosfito de potássio por 20 dias, enquanto os demais ficaram menos dias expostos à ação do fosfito, resultando em maior severidade da PBE nas mudas (Tabela 2).

Aos 21 dias de avaliação, observou-se que o $\mathrm{T}_{3}$ (2 aplicações aos 4 e 2 dias antes da inoculação) se equiparou aos tratamentos $T_{1}$ e $\mathrm{T}_{2}$, os quais apresentaram menor severidade em relação aos demais tratamentos, provavelmente, devido ao maior tempo de ação do fosfito, no caso $\mathrm{T}_{1}$ e $\mathrm{T}_{2} 27$ dias, e $\mathrm{T}_{3}$ com 25 dias de ação do fosfito na doença. Aos 28 dias de avaliação, as mudas em que se aplicaram os tratamentos $\mathrm{T}_{1}, \mathrm{~T}_{2}$ e $\mathrm{T}_{3}$ apresentaram menor severidade à doença em relação aos demais tratamentos, enquanto os tratamentos $\mathrm{T}_{5}$ e $\mathrm{T}_{6}$ favoreceram a maior severidade da PBE nas mudas de pupunheira. Isso indica que quanto antes forem realizadas as aplicações preventivas do fosfito de potássio, maior será o controle da doença. Observaram-se diferenças significativas da severidade entre as épocas de avaliação de 7 aos 14 dias nos tratamentos $\mathrm{T}_{4}, \mathrm{~T}_{5} \mathrm{e} \mathrm{T}_{6}$. Entre 14 aos 21 dias, houve diferenças significativas nos tratamentos $T_{5}$ e $\mathrm{T}_{6}$. Entretanto, entre 21 dias e 28 dias, não houve diferenças significativas entre as épocas de avaliação. Os tratamentos $T_{1}, T_{2}$ e $T_{3}$ não tiveram interação com as épocas de avaliação. Entre 21 e 28 dias de avaliação, verificou-se uma tendência de estabilidade na severidade da doença, quando atingiu próxima a nota máxima da escala descritiva (nota 4 - planta morta) nos tratamentos onde não houve o efeito do fosfito no controle da PBE.

Os tratamentos $\mathrm{T}_{1} \mathrm{e} \mathrm{T}_{2}$ na aplicação preventiva de fosfito apresentaram severidade média inferior aos demais tratamentos, cuja incidência da doença não ultrapassou $20 \%$.

Jackson et al. (2000) relataram que aplicações foliares de fosfito, 48 horas antes da inoculação de P. cinnamomi, diminuíram o desenvolvimento da podridão de raiz em eucalipto. Segundo estes autores, houve aumento na concentração de enzimas de defesa produzidas pela planta. $\mathrm{O}$ fosfito contribuiu para a redução da produção de zoósporos de $P$. cinnamomi em mudas de eucaliptos, mas os zoósporos remanescentes foram capazes de infectar material vegetal. Assim, o fosfito pode desacelerar a produção, mas não impedir a disseminação de $P$. cinnamomi (WILKINSON et al., 2001).

No Experimento III, houve interação entre os fatores (dosagens x número de aplicações), sendo que, na dosagem de 2,5 mL.L-1 (Tabela 3), quando se realizam duas aplicações de fosfito de potássio, não se observa redução da severidade da PBE, enquanto ao realizar três aplicações há redução da severidade da PBE. No tratamento com a dosagem de 5,0 mL.L1 , observa-se o controle da PBE independentemente do número de aplicações, reduzindo a severidade da doença, enquanto o tratamento que continha somente água não mostrou resposta, como já era esperado.

Pela Tabela 3, nota-se que, na aplicação do fosfito de potássio na dosagem de 2,5 mL.L $\mathrm{L}^{-1}$, houve diferenças significativas entre o número de aplicação; entretanto, na dosagem de 5,0 mL.L $\mathrm{L}^{-1}$, não houve diferenças significativas com o número de aplicações. $\mathrm{Na}$ aplicação do fosfito de potássio na dosagem de 2,5 mL.L $\mathrm{L}^{-1}$ com três aplicações e na dosagem de 5,0 $\mathrm{mL} \cdot \mathrm{L}^{-1}$ com duas ou três aplicações, houve redução da severidade da PBE. De forma geral, a incidência da doença em mudas tratadas com fosfito de potássio atingiu $100 \%$ em todos os tratamentos.

No presente trabalho, constatou-se que fosfito de potássio, quando aplicado de forma preventiva em mudas de pupunheira, foram mais efetivos em conter a incidência e a severidade, mas não preveniu a infecção pelo oomiceto P. palmivora.

$\mathrm{O}$ efeito direto do fosfito no metabolismo de Phytophthora carece de investigação, mas o fosfito 
de potássio mostrou-se promissor na supressão da PBE, atuando na maior parte dos casos na forma de prevenção, ou seja, antes da inoculação, em ambas as doses testadas, diferindo na quantidade de pulverização.

Segundo Dianese et al. (2009), duas aplicações por semana de fosfito de potássio, fosfito de cálcio ou fosfito de magnésio por três semanas consecutivas, antes da inoculação do patógeno $P$. palmivora, reduziram a intensidade da doença no mamoeiro. Os resultados obtidos no experimento I diferem dos observados pelo autor supracitado, pois verificou-se que apenas o fosfito de potássio foi eficiente para o controle da doença em mudas de pupunheira.

TABELA 1- Tipos de fosfito, severidade nas formas de aplicação e as respectivas incidências da podridão da base do estipe ocorridas aos 28 dias após a inoculação da suspensão de zoósporos de Phytophthora palmivora (Experimento I - Colombo-PR/2011).

\begin{tabular}{cccc}
\hline \multirow{2}{*}{ Tipos de Fosfito } & \multicolumn{2}{c}{ Severidade nas formas de aplicação } & Incidência \\
\cline { 2 - 3 } & Preventivo & Curativo & $(\%)$ \\
\hline Potássio & $3,2 \mathrm{aA}$ & $3,8 \mathrm{aB}$ & 100 \\
Cálcio & $3,9 \mathrm{bA}$ & $4,0 \mathrm{aA}$ & 100 \\
Zinco & $4,0 \mathrm{bA}$ & $4,0 \mathrm{aA}$ & 100 \\
Magnésio & $3,8 \mathrm{bA}$ & $4,0 \mathrm{aA}$ & 100 \\
Manganês & $3,8 \mathrm{bA}$ & $4,0 \mathrm{aA}$ & 100 \\
Testemunha & $4,0 \mathrm{bA}$ & $4,0 \mathrm{aA}$ & 100 \\
\hline $\mathrm{CV}(\%)$ & \multicolumn{2}{c}{10,33} \\
\hline
\end{tabular}

Médias seguidas pela mesma letra minúscula na coluna e maiúscula na linha não diferem entre si, pelo teste de Tukey, a $5 \%$ de probabilidade.

TABELA 2- Número de aplicações, severidade nas respectivas épocas de avaliação, área abaixo da curva de progresso da doença (AACPD) e as respectivas incidências da podridão da base do estipe após a inoculação de suspensão de zoósporos de Phytophthora palmivora (Experimento II Colombo- PR/2011).

\begin{tabular}{|c|c|c|c|c|c|c|}
\hline \multirow{2}{*}{ Número de aplicações } & \multicolumn{4}{|c|}{ Severidade nas épocas de avaliação } & \multirow{2}{*}{ AACPD } & \multirow{2}{*}{ Incidência (\%) } \\
\hline & 7 dias & 14 dias & 21 dias & 28 dias & & \\
\hline $\mathrm{T} 1^{*}$ & $0,0 \mathrm{a} A$ & $0,1 \mathrm{aA}$ & $0,5 \mathrm{aA}$ & $0,5 \mathrm{aA}$ & 5,95 & 20 \\
\hline $\mathrm{T} 2$ & $0,0 \mathrm{a} A$ & $0,1 \mathrm{aA}$ & $0,3 \mathrm{aA}$ & 0,3 a A & 3,85 & 20 \\
\hline $\mathrm{T} 3$ & $0,6 \mathrm{a} A$ & $1,1 \mathrm{bA}$ & $1,0 \mathrm{a} A$ & $1,2 \mathrm{bA}$ & 21,00 & 60 \\
\hline $\mathrm{T} 4$ & $0,3 \mathrm{a} A$ & $1,6 \mathrm{bB}$ & $2,4 \mathrm{bBC}$ & $2,6 \mathrm{cC}$ & 38,15 & 100 \\
\hline T5 & $0,4 \mathrm{a} \mathrm{A}$ & $1,8 \mathrm{bcB}$ & $2,9 \mathrm{bcC}$ & $3,2 \mathrm{cdC}$ & 45,50 & 100 \\
\hline T6 & $0,8 \mathrm{a} A$ & $2,6 \mathrm{cB}$ & $3,8 \mathrm{cC}$ & $3,9 \mathrm{dC}$ & 61,25 & 100 \\
\hline
\end{tabular}

* T1 - 3 aplicações de fosfito de potássio aos 6; 4 e 2 dias antes da inoculação; T2 - 2 aplicações de fosfito de potássio aos 6 e 4 dias antes da inoculação; T3 - 2 aplicações de fosfito de potássio aos 4 e 2 dias antes da inoculação; T4 - Uma aplicação de fosfito de potássio aos 2 dias antes da inoculação; T5 - Uma aplicação de fosfito de potássio no mesmo dia da inoculação; e T6 - Testemunha, com inoculação e pulverização no mesmo dia, somente com água.

Médias seguidas pela mesma letra minúscula na coluna e maiúscula na linha não diferem entre si, pelo teste de Tukey, a 5\% de probabilidade. 
TABELA 3- Dosagens de fosfito de potássio, número de aplicações e as respectivas incidências da podridão da base do estipe observada aos 28 dias após a inoculação da suspensão de zoósporos de Phytophthora palmivora (Experimento III - Colombo-PR/2011).

\begin{tabular}{cccc}
$\begin{array}{c}\text { Dosagens de fosfito } \\
\text { de potássio }\end{array}$ & \multicolumn{4}{c}{ Aplicações de fosfito de potássio Incidência (\%) } \\
& Duas & Três & 100 \\
\hline $2,5 \mathrm{~mL} \cdot \mathrm{L}^{-1}$ & $3,1 \mathrm{bB}$ & $1,8 \mathrm{aA}$ & 100 \\
$5 \mathrm{~mL} \cdot \mathrm{L}^{-1}$ & $1,3 \mathrm{aA}$ & $1,3 \mathrm{aA}$ & 100 \\
\hline Somente água & $3,5 \mathrm{bA}$ & $3,5 \mathrm{bA}$ & \\
\hline $\mathrm{CV}(\%)$ & \multicolumn{3}{c}{31,7}
\end{tabular}

Duas aplicações de fosfito de potássio aos 4 e 6 dias antes da inoculação da suspensão de zoósporos de $P$. palmivora Três aplicações de fosfito de potássio aos 2; 4 e 6 dias antes da inoculação da suspensão de zoósporos de $P$. palmivora Médias seguidas pela mesma letra minúscula na coluna e maiúscula na linha não diferem entre si, pelo teste de Tukey, a 5\% de probabilidade.

\section{CONCLUSÕES}

1- Entre os fosfitos testados, o fosfito de potássio apresenta menor severidade da PBE em mudas de pupunheira.

2- A dosagem de $2,5 \mathrm{~mL} . \mathrm{L}^{-1}$ de fosfito de potássio reduz a severidade da PBE quando aplicado 3 vezes (2;4 e 6 dias antes da inoculação), e a dosagem de 5,0 mL. $\mathrm{L}^{-1}$ reduz a severidade, independentemente do número de aplicações testada.

3- O modo de aplicação do fosfito de potássio, quando realizada com maior antecedência à inoculação com Phytophthora palmivora, reduz os sintomas da doença, enquanto não há redução significativa da incidência e da severidade da PBE quando fosfito de potássio é aplicado de forma curativa, independentemente da dosagem ou do número de aplicações.

4- A melhor época de avaliação da severidade da PBE é aos 21 dias após a inoculação da Phytopthora palmivora.

\section{REFERÊNCIAS}

ARAÚJO, L.; STADNIK, M. J.; BORSATO, L. C.; VALDEBENITO-SANHUEZA, R. M. Fosfito de potássio e ulvana no controle da mancha foliar da gala da macieira. Tropical Plant Pathology, Brasília, v.33, n. 2, p. 148-152, 2008.

BLUM, L. E. B.; AMARANTE, C. V. T.; DEZANET, A.; LIMA, E. B.; HACK NETO, P.; ÁVILA, R. D.; SIEGA, V. Fosfitos aplicados em pós-colheita reduzem o mofo-azul em maçãs 'Fuji' e 'Gala'. Revista Brasileira de Fruticultura, Jaboticabal, v. 29, n. 2, p. 265-268, 2007.
CAMPBELL, C.L.; MADDEN, L.V. Introduction to plant disease epidemiology. New York: Wiley, 1990.

DANIEL, R.; GUEST, D. Defence responses induced by potassium phosphonate in Phytophtora palmivora challenged Arabidopsis thaliana. Physiological-andMolecular-Plant-Pathology, Oxford, v.67, n.3-5, p.194-210, 2006.

DIANESE, A. C.; BLUM, L.E.B.; DUTRA, J.B.; LOPES, L.F. Aplicação de fosfito de potássio, cálcio ou magnésio para a redução da podridão-do-pé do mamoeiro em casa de vegetação. Ciência Rural, Santa Maria, v.39, n.8, p.2309-2314, 2009.

DIANESE, A. C.; BLUM, L.E.B.; DUTRA, J.B.; LOPES, L.F.; SENA, M. C.; FREITAS, L. F. Avaliação do efeito de fosfitos na redução da varíola (Asperisporium caricae) do maoeiro (Carica papaya). Revista Brasileira de Fruticultura, Jaboticabal, v. 30, n. 3, p. 834-837, 2008.

GENTILE, S.; VALENTINO, D.; TAMIETTI, G. Control of Ink disease by trunk injection of potassium phosphate. Journal of Plant Pathology, Wageningen, v.3, n.91, p. 565-571, 2009.

JACKSON, T.J.; BURGESS, T.; COLQUHOUN, I.; HARDY, G.E. Action of the fungicide phosphate on Eucalyptus marginata inoculated with Phytophtora cinnamomi. Plant Pathology, Oxford, v.49, n.1, p.147-154, 2000. Disponível em: $<\underline{\text { http: } / /}$ www3.interscience.wiley.com/journal/119182580/ abstract?CRETRY $=1 \&$ STRETRY $=0>$. Acesso em: 20 jan. 2012.

FERREIRA, D.F. Sisvar 4.6 sistema de análise estatísticas. Lavras: UFLA, 2003. 
MORA-URPI, J.; WEBER, J.C.; CLEMENT, R.C. 1997. Peach palm (Bactris gasipaes Kunth). Promoting the conservation and use of funder utilized and neglected crops, 20. Gatersleben: Institute of Plant Genetics and Crop Plant Research; Roma: IPGRI, 1997.83p.

SANTOS, A.F. do; LUZ, E.D.M.N.; FIANTO, P.D.; TESSMANN, D.J.; VIDA, J.B. Primeiro relato da podridão do estipe da pupunheira causado por Phytophthora palmivora, no Estado do Paraná. Fitopatologia Brasileira, Brasília, v.30, p.81-84, 2004.
WILKINSON, C.J.; HOLMES, J.M.; DELL, B.;TYNAN, K.M.; McCOMB, J.A.; SHEARER, B.L.; COLQUHOUN, I.J.; HARDY, G.E.St.J. Effect of phosphite on in planta zoospore production of Phytophthora cinnamomi. Plant Pathology, Oxford, v.50, p.587-593, 2001. 